\title{
Changes in stress levels and the immuno-modulatory effects of Echinaforce $\mathbb{R}$
}

\author{
V. Wong ${ }^{1}$, A. Ravishankar ${ }^{2}$, A. Gilmour ${ }^{3}$, S. E. E. Gates ${ }^{4}$, J. McNulty $^{5}$, A. Pritchard ${ }^{5}$, H. Cargill ${ }^{6}$, \\ R. Schoop ${ }^{7}$ S. Ramnarine ${ }^{7}$ and M. R. Ritchie ${ }^{5,6}$ \\ ${ }^{1}$ University of Aberdeen, Aberdeen AB24 3FX, UK, ${ }^{2}$ University of London, London WC2R $2 L S$, UK, ${ }^{3}$ University of \\ Manchester, Manchester M13 9PL, UK, ${ }^{4}$ Cambridge University, Cambridge CB2 ITN, UK, ${ }^{5}$ Arbroath High School, \\ Arbroath DDI1 3BN, UK, ${ }^{6}$ Napier University, Edinburgh EH14 1DJ, UK and ${ }^{7}$ University of Edinburgh, \\ Edinburgh EH8 9YL, UK
}

\begin{abstract}
Stress, which is a huge occupational health problem in the UK, can weaken the immune system, leaving individuals more susceptible to various infections ${ }^{(1)}$. Several studies have shown Echinacea purpurea (a medicinal plant) to be beneficial in treating and preventing influenza-type infections and the common cold ${ }^{(2,3)}$. However, to date little is known about the effects of Echinacea purpurea on the immune response in individuals whose stress levels change. Using an ex vivo stimulation model the present study aimed to investigate the immuno-modulatory effects of repeated Echinaforce ( ) (extract of Echinacea purpurea; Bioforce AG, Roggwil, Switzerland) dosing in a heterogenous group of healthy subjects during and after a period of stress.

Thirty healthy subjects (age range 18-57 years, mean 20.6 years) with high perceived stress levels (measured using a perceived stress score-10 (PSS-10) questionnaire ${ }^{4}$ ) enrolled in phase I of the study. Daily blood samples collected throughout the $10 \mathrm{~d}$ study period were analysed for the anti-inflammatory mediator IFN- $\gamma$ after ex vivo stimulation by lipopolysaccharide (LPS; variant O55:B5 from Escherichia coli; $100 \mathrm{ng} / \mathrm{ml}$ ) and super-antigen staphylococcal enterotoxin B (SEB; $25 \mathrm{ng} / \mathrm{ml})$. After $2 \mathrm{~d}$ of baseline measurements treatment was started with $4 \times 1 \mathrm{ml}$ Echinaforce $\mathbb{B} / \mathrm{d}$ (low dose) for $5 \mathrm{~d}$ and subsequently increased to $10 \times 1 \mathrm{ml} / \mathrm{d}$ (high dose) for $4 \mathrm{~d}$. After $5 \mathrm{weeks}$ all subjects completed another PSS-10 questionnaire. Eight subjects whose perceived stress level had dropped by more than five scores completed phase II, in which the procedures used in phase I were repeated.

Concentrations of IFN- $\gamma$ showed a marked increase during 'low-dose' Echinaforce $\mathbb{B}$ treatment in phase I (stress period; mean PSS-10 score 19.1; SD 7.6) and remained higher than baseline production throughout the treatment period. Volunteers also experienced a significant transient increase in IFN- $\gamma(>25 \%)$ with peak induction of $50 \%(P<0.05)$ on the first day of 'high-dose' treatment and a subsequent fall to baseline at the last day of treatment. This effect may have resulted in a reduction in volunteers' susceptibility to colds as a result of the antiviral property of IFN- $\gamma$. Interestingly, this effect was not observed in the same subjects whose perceived stress dropped by more than five scores in phase II (non-stress period; mean PSS-10 score 12.0; SD 5.0).

The results demonstrate a very specific adapted immune-modulatory activity of Echinaforce $\mathbb{R}$. As advantageous effects were only seen when volunteers were perceived to be stressed, it can be hypothesised that Echinaforce $\mathbb{R}$ treatment may have a protective effect against infections during periods of stress. Although the volunteers who participated in both phases act as their own control, a placebo controlled study will be beneficial in further confirming the findings.
\end{abstract}

This study was funded by Bioforce AG, Roggwil, Switzerland.

1. Schedlowski M \& Schmidt RE (1996) Naturwissenschaften 83, 214-220.

2. Blumenthal M, Milot B \& Oliff HS (2007) HerbalGram 74, 28-31.

3. Woelkart K, Linde K \& Bauer R (2008) Planta Med 74, 633-637.

4. Cohen S, Kamarck T \& Mermelstein R (1983) J Health Soc Behav 24, 385-396. 